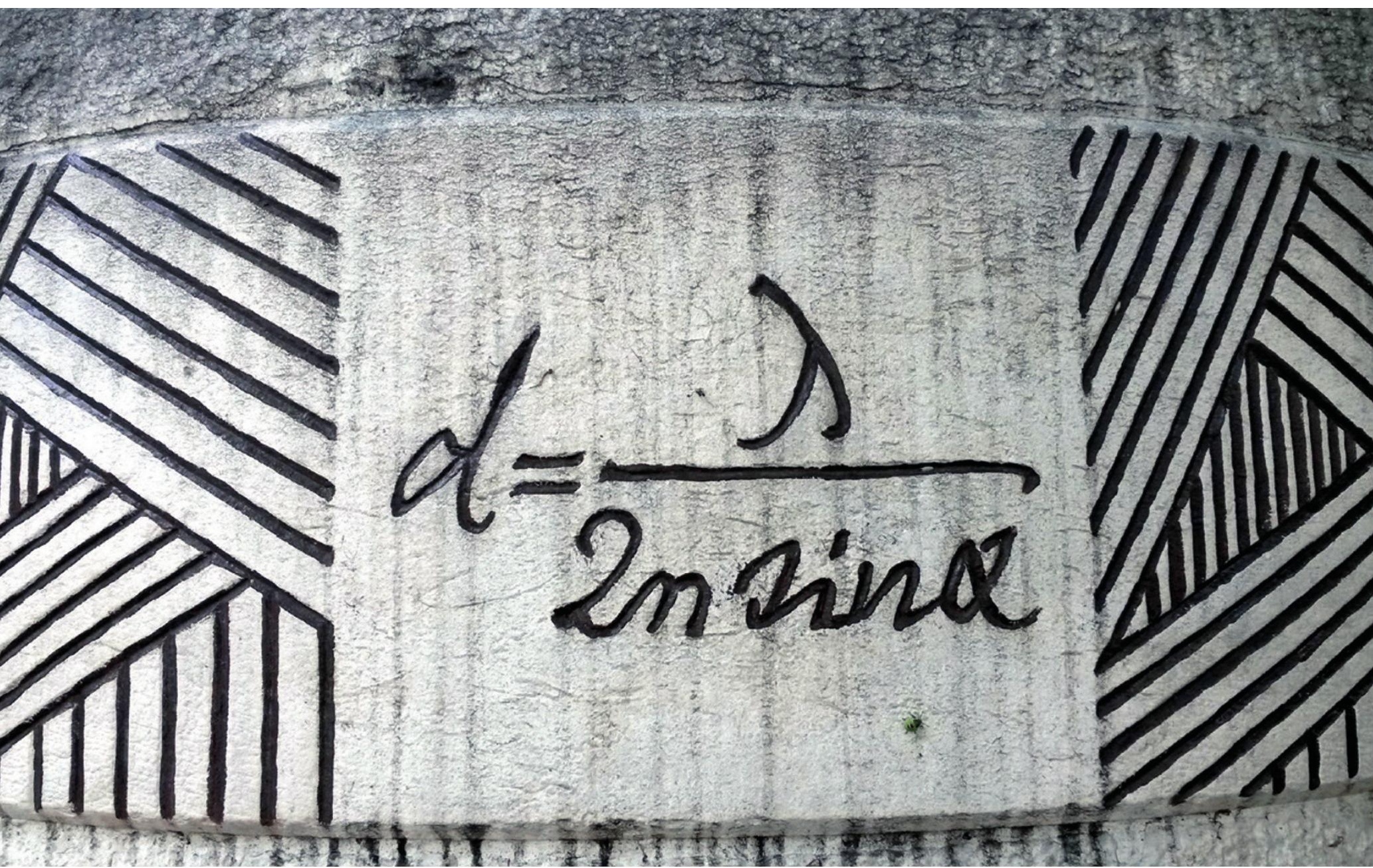

The equation describing the diffraction limit is engraved on a monument to optical physicist Ernst Abbe in Jena, Germany.

CELL IMAGING

Beyond the limits

Powerful super-resolution microscopes that allow researchers to explore the world at the nanoscale are set to transform our understanding of the cell.

\section{KATHERINE BOURZAC}

$\mathrm{D}$ yche Mullins dons a pair of Oculus Rift $3 \mathrm{D}$ goggles and is transported from a cubicle at the University of California, San Francisco, into a virtual world. Through the virtual-reality headset, Mullins watches an immune cell that appears to be the size of a child. The cell crawls through a maze of collagen fibres. Mullins can move around the image, 'pushing' in so that the neutrophil is crawling directly above him, or turn the image around to watch the cell's motions from different angles.

This is not a simulation. The video was made using a cutting-edge microscope invented by Eric Betzig, an engineer at the Howard Hughes
Medical Institute's Janelia Research Campus in Ashburn, Virginia. Foundational work in cell migration is based on studies done in the 1950s and 1960s in 2D, at low resolution and on glass slides. Seeing cells more clearly, says Mullins, who is a molecular biologist, is helping to overturn received wisdom about the fundamentals of how cells move, whether they are immune cells moving through tissue or amoebae in a pond. With new imaging tools, he can watch their movement in vivid detail.

These tools are redefining microscopy's frontier, and their potential impact on biology is huge. Until the development of super-resolution microscopes, which began in 2000, life's underlying molecular world appeared as a blur, only hinted at by relatively low-resolution light

microscopy or captured in crisp, but static single-frame electron micrographs. "Now we have the proof that these barriers can be overcome," says Stefan Hell, director at the Max Planck Institute for Biophysical Chemistry in Göttingen, Germany. "We're just at the beginning of what's possible." Hell, a newly minted Nobel laureate, gave a lecture on the subject this year at the 65th Lindau Nobel Laureate Meeting in Germany, rousing excitement among his fellow laureates and the young scientists in attendance.

Hell was jointly awarded the Nobel Prize

\section{$\rightarrow$ NATURE,COM}

For an animation about

Stefan Hell and his work

visit: go.nature.com/hhrbee in Chemistry in 2014 with Betzig and William Moerner for their development of 
super-resolved fluorescence microscopy. Before their work, the nanoscale details of life in motion went unwitnessed. That has been a big blind spot. Life is dynamic: cells not only crawl around, like Mullins' neutrophil, they divide, shuttle chemicals from one internal structure, or organelle, to another, and so much more. Aside from cell migration, researchers are using super-resolution microscopes to look at what happens when a group of viruses attacks a cell in real time, and they are turning these instruments on the chemical connections between neurons in the brain. "This technology will be transformative," says Tomas Kirchhausen, a cell biologist at Harvard Medical School in Boston, Massachusetts, and another early adopter of these tools. "I'm ready to put my other instruments on eBay."

\section{A SHARPER IMAGE}

Hell was the first to break the existing limits on light microscopes. But not too long ago, he told the Lindau audience, no one took him seriously (a common remark made by Nobel prize winners). Ideas about the limits of imaging were not only set in scientists' minds, but literally set in stone. On the lecture screen, Hell showed a photograph of a monument to one of his scientific forebears, Ernst Abbe, in Jena, Germany - a stone engraved with the equation describing the diffraction limit.

Abbe rose from humble beginnings to become a brilliant optical physicist at German microscope manufacturer Zeiss. He was the first to understand how images are made in light microscopes, explained Hell, and he was able to make the best instruments. Abbe figured out that when a beam of light is focused on a spot whose size is smaller than about half its wavelength, it starts to interfere with itself, and the image becomes blurred. If two objects are closer together than that distance - a couple of hundred nanometres - they cannot be resolved under a conventional light microscope no matter how perfect the lens. This is Abbe's diffraction limit.

It is in large part thanks to Abbe's work that light microscopes have been powerful research tools. "I have huge respect for Abbe," says Hell, who named his own microscopy company, based in Göttingen, Germany, Abberior. Abbe was not wrong, he was a product of his time. In 1873, when he published his work on the diffraction limit, the molecule was an unproven idea and making better microscopes was all about developments in optical science.

Hell realized that chemistry could take things further. Below the diffraction limit is a rich microbiological world that includes viruses, proteins and the fine details of organelles such as the energy-generating mitochondria. Hell was determined to bring this world to light, and became obsessed with breaking the diffraction limit. He focused on a technique called fluorescence microscopy, a common tool used by biologists. The method

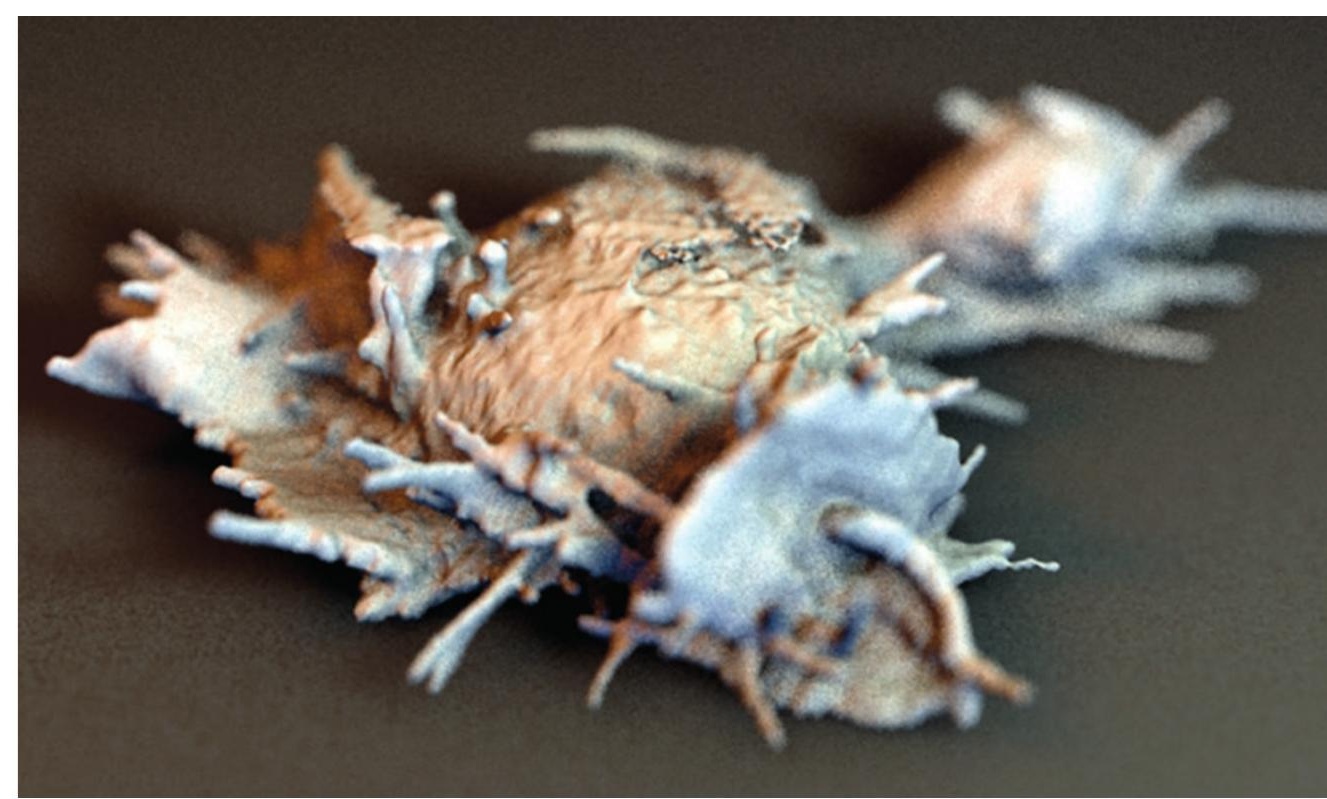

A live cell captured by a type of microscopy developed by Nobel prizewinner Eric Betzig.

is a form of light microscopy, augmented by fluorescent chemical labels. These bright red, green or other coloured tags attach to the specific molecules that a biologist is interested in and act as beacons to help locate particular types of cells or structures within cells.

In the 1990s, Hell read an article about a quantum mechanical phenomenon called stimulated emission - a way to control the fluorescence of these labels - and was "electrified". He realized that imaging could be about more than just great lenses - he could use chemistry to get beyond the diffraction limit.

Hell designed a system that used two beams of light, one to stimulate fluorescent molecules, and another that immediately turned most of them off in such a way that only those at the centre of the light beam continued to shine ${ }^{1}$. The technique, which he called stimulated emission depletion (STED) microscopy, does not break the diffraction limit but, as Hell puts it, plays a game to get around it. The resulting cylindrical beam - shaped like the outline of a donut with the inner circle filled in - is then scanned over the sample to get the full picture (see 'Under the microscope'). Hell's first superresolution microscope in 2000 only exceeded the diffraction limit by around a factor of two, but it brought the barrier down.

Hell is a self-described dreamer, and felt shut out of the mainstream of science before his Nobel-winning discovery. Whereas Betzig cultivates the aura of a cranky outsider, and in his talk at Lindau this year, he made it clear that he has no patience for the strictures of academia. Betzig calls himself a "tool-builder" - an engineer who wants to build the best microscopes possible.

Betzig was also long-obsessed with surpassing Abbe's diffraction limit. Working on near-field microscopy at renowned research centre Bell Labs in Murray Hill, New Jersey, in the early 1990s, he made advances on a technology called near-field imaging. But he grew frustrated with research on what he did not consider to be a very useful tool. Near-field microscopy technically beats the diffraction limit, but it is not very practical because it uses a sharp imaging tip that must be extremely close to the sample. Betzig left research for several years and worked at his father's company, designing machine-tooling equipment. After inventing a design that the company could not sell, he had what he calls his second midlife crisis. He kept up-to-date with the scientific literature, and reading about green fluorescent proteins brought him back to science - into

the same sphere in

"The technology will be which Moerner was making waves - and so he returned to the problem of the diffraction limit.

Like Hell's solution, Betzig's relies on chemistry, in this case a phenomenon called photoswitching that Moerner was studying ${ }^{2}$. Working at IBM in San Jose, California, Moerner was the first to measure the absorption spectrum of a single molecule ${ }^{3}$. Then, in 1997, he went on to show that certain fluorescent molecules could be turned on and off with a beam of light. The underlying physics are different from STED, but the implication is the same: being able to turn fluorescent molecules on and off as though they were light bulbs.

Hell's microscopes raster a narrow beam over a sample to fill in the image zone by zone, whereas Betzig's work combines several faint snaps of the whole imaging zone to make a complete picture. In the latter case, before each image capture, the microscope illuminates 


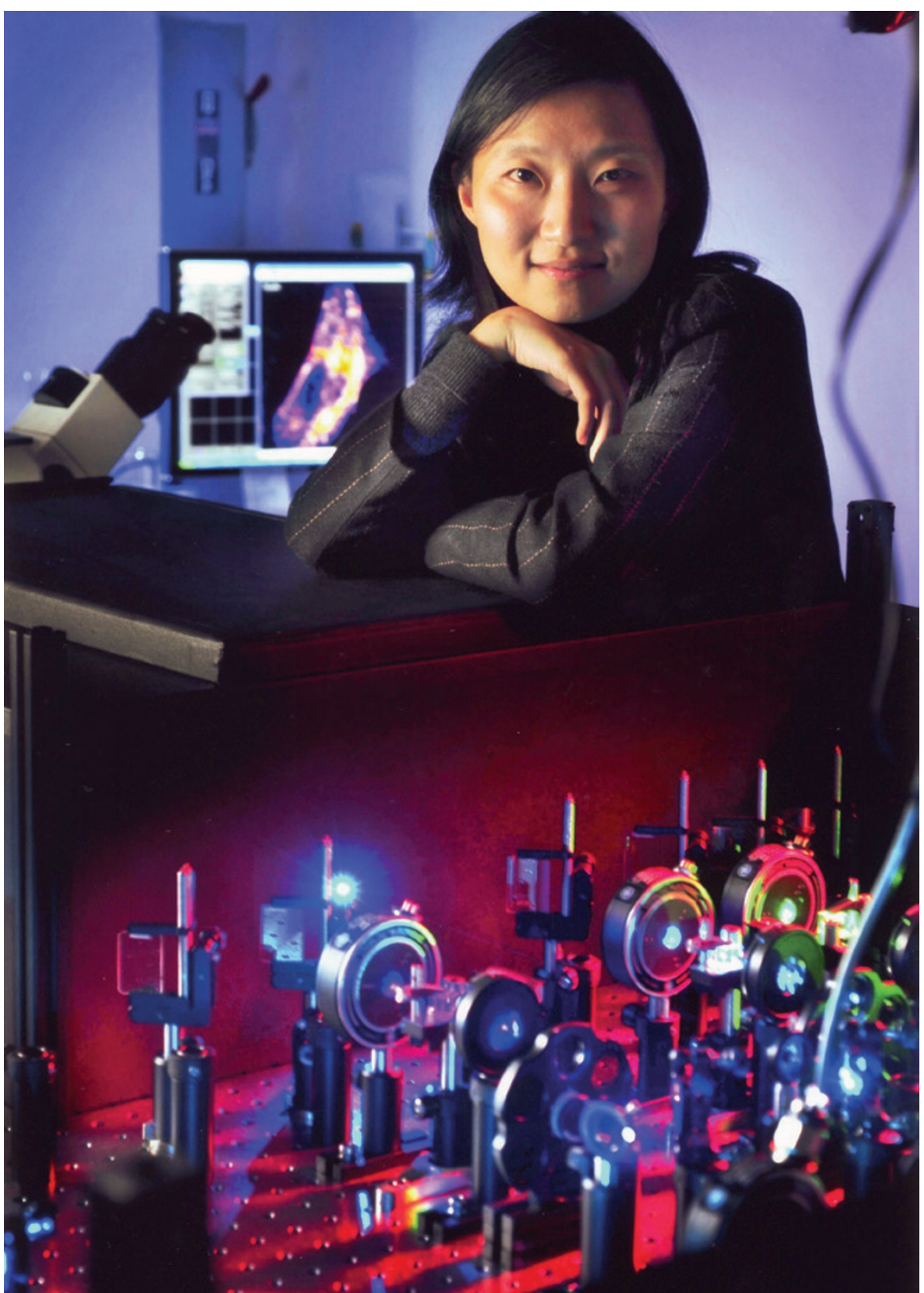

Xiaowei Zhuang developed the super-resolution microscopy method STORM.

the sample with a weak beam of light that only turns on a small fraction of the fluorescent molecules in the sample. This is repeated again and again until all of the molecules have been located. Even if two molecules are closer together than the diffraction limit, they will appear in different sub-images, so they can all be seen in composite.

\section{CELLS IN FOCUS}

Biologists trying out the new technologies are amazed by what is possible. In the seventeenth century, before he examined a slice of cork that he had put under his microscope, Robert Hooke did not know that cells existed - no one did. But there they were: “... indeed the first microscopical pores I ever saw, and perhaps, that were ever seen, for I had not met with any Writer or Person, that had made any mention of them before this," he wrote in 1665. Stories from early users of super-resolution imaging are reminiscent of Hooke. "Super-resolution is not just about getting sharper pictures," says Xiaowei Zhuang, a biophysicist at Harvard University in Cambridge, Massachusetts, who developed another super-resolution method called Stochastic Optical Reconstruction Microscopy (STORM), which she and her colleagues published in 2006 (ref. 4). "You can discover things that have never been seen before."

Using STORM to look at the structural protein actin in neurons in 2012, she saw something surprising and new. Zhuang is interested in synapses, the electrochemical connections between neurons that are the basic units of brain circuits. She had asked her postdoctoral researchers to image the structure of synapses by mapping out actin at high resolution. But the most noticeable feature of the pictures turned out to lie next to the synapses: in the images, the axons - the slender projections that transmit neurons' outgoing signals looked odd.

All along the axons, the team saw regularly spaced rings of actin. "It's a remarkable periodic structure - every 180 nanometres, an actin ring," says Zhuang. "It's beautifully laid out, it almost looks like the structure of a crystal." The features of this pattern are under the diffraction limit, and so the rings had been previously invisible. Under a conventional fluorescent microscope you can see a smear indicating the presence of actin, but the periodic rings, so distinct under Zhuang's STORM microscope, are a blur. Nor had anyone noticed the rings under an electron microscope because other filamentous structures in the cell obscure them, she says.

Zhuang has found that the actin rings are present in all axons, but only in patches on a small fraction of dendrites, the neural projections that receive signals. The 'axonal skeleton' helps axons to maintain their mechanical stability and conduct impulses. This understanding is supported by other studies ${ }^{5}$, which show that worms genetically engineered to lack the protein that connect the rings have fragile axons, impaired movement and a reduced response to mechanical stimuli.

Harvard's Kirchhausen has had similarly eye-opening moments using a microscope built with Betzig, which uses thin sheets of light to illuminate one planar slice of a sample at a time and image it using Betzig's super-resolution method. It is the most recent example of Betzig's work. Kirchhausen has spent decades studying the formation of membrane bubbles called vesicles that are just 30-80 nanometres in diameter. Vesicles carry chemicals around the cell and also help the cell engulf, transport and break down unwanted molecules and microbes. In 2004, Kirchhausen completed the first image of the structure of the protein complex that pinches off vesicles from a membrane ${ }^{6}$. He used electron microscopy to generate images with near-atomic resolution, going down to 0.8 nanometres. But

"Super-resolution is not just about getting sharper pictures. You can discover things that have never been seen before." although he was making progress in determining the structure of vesicles, he says, "I realized I should be paying attention to the dynamics." Using one of Betzig's machines, he was finally able to watch the process. Over a period of 30-90 seconds, hundreds of molecules assemble into a kind of geodesic dome that pulls out a bubble of membrane. Then they all fall away to leave the vesicle. Kirchhausen can now watch 


\section{UNDER THE MICROSCOPE}

Our understanding of cells such as neurons has benefited from advances in imaging. By Katherine Bourzac.

\section{LIGHT MICROSCOPY SIXTEENTH CENTURY}

The first light microscopes used relatively simple arrangements of lenses to magnify tissue; later versions used external illumination and stains to make features even clearer. The original inventor is disputed, but the first microscopes are thought to have emerged from work on telescopes.

\section{ELECTRON MICROSCOPY 1933}

Physicist Ernst Ruska created the first microscope to form an image by bouncing beams of electrons off a sample. He shared the 1986 Nobel Prize in Physics for this work. Electron microscopes can resolve atomic-scale detail - as static, structural images - because the wavelength of an electron is much shorter than that of light.

\section{FLUORESCENCE MICROSCOPY 1990s}

This technique allows researchers to locate parts of the cell using chemistry. Molecules of interest can be labelled by attaching fluorescent tags to proteins, and gene expression can be monitored by inserting a fluorescent protein gene into the genome (a method recognized by the Nobel Prize in Chemistry in 2008).

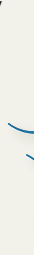

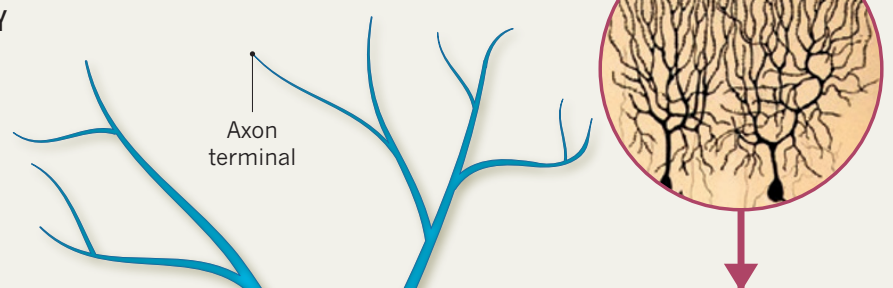

Under a light microscope, neuroscientist Santiago Ramón y Cajal saw the delicate branching of dendrites. He shared the Nobel Prize in Physiology or Medicine in 1906 with Camillo Golgi for their work on the structure of the nervous system.

SUPER RESOLUTION 2000 onwards

The latest microscopes take resolution to the nanoscale. The subject of the 2014 Nobel Prize in Chemistry, super-resolution microscopy zooms in on individual molecules and subcellular structures by computationally combining many fluorescence images. Stimulated emission depletion, or STED, microscopy is an example of this technique.

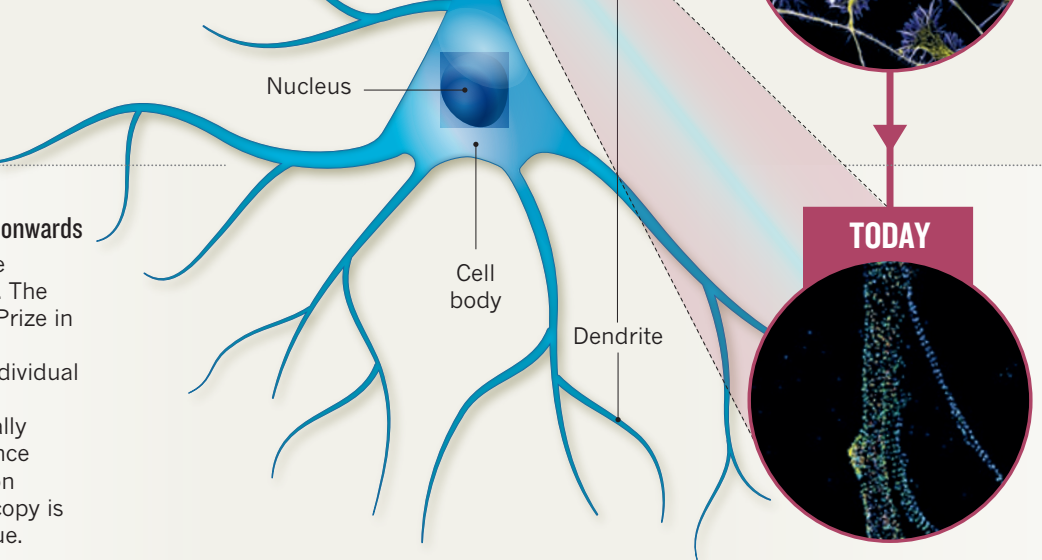

Electron microscopes revealed the branching or 'spines' on the dendrites of neurons for the first time. In 1959, Edward George Gray published images of dendritic spines and established that the connections between neurons (synapses) occur at these sites.

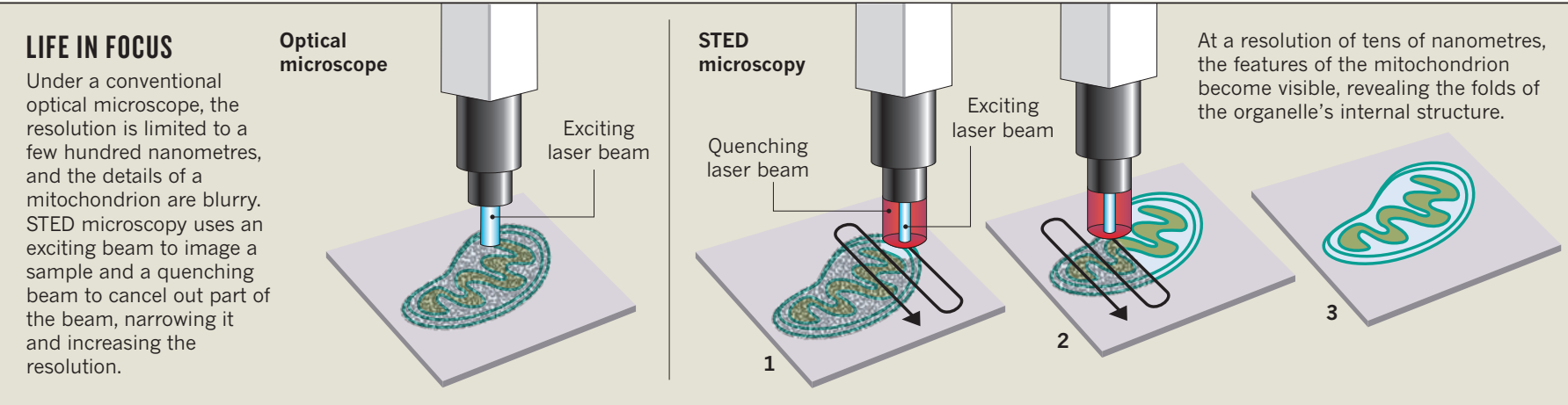




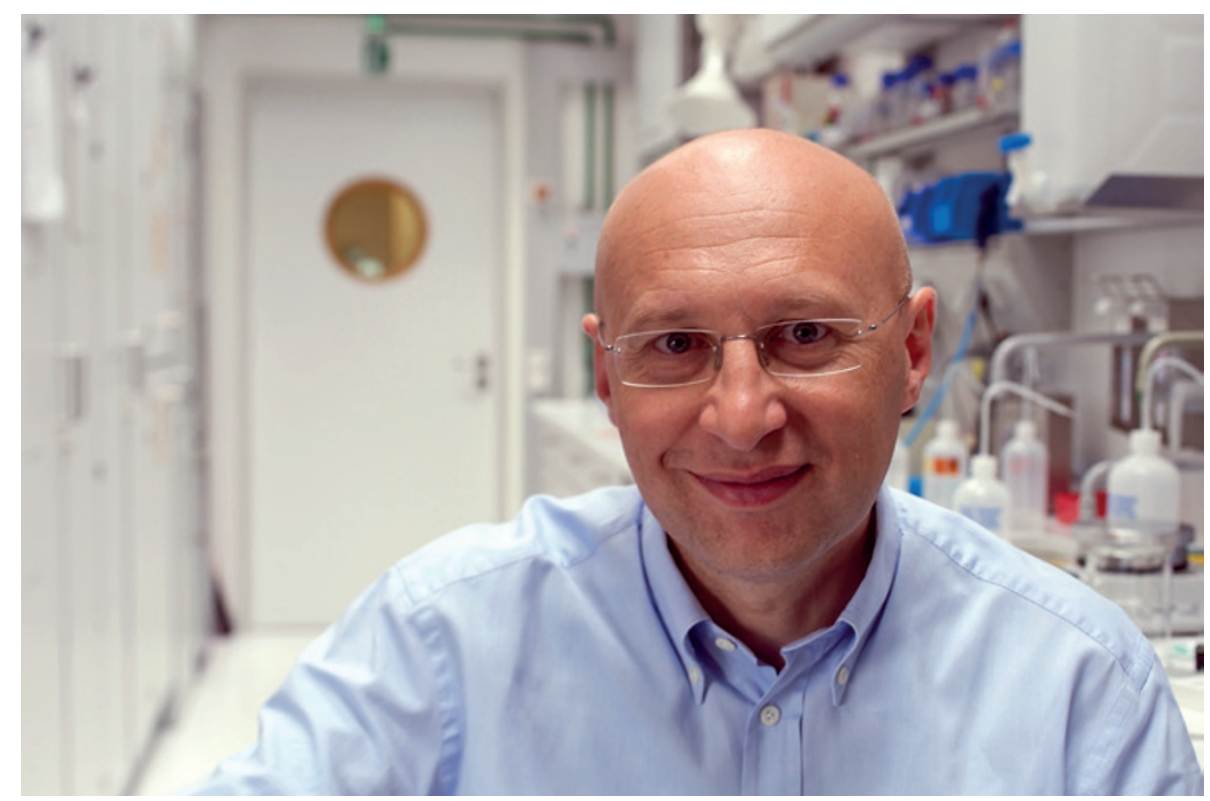

Stefan Hell was jointly awarded the Nobel prize for his work on super-resolution microscopy.

this happen thousands of times a minute, throughout a cell.

As soon as the super-resolution microscope was up and running in 2014, Kirchhausen started using it for various other projects requiring super-resolution in $3 \mathrm{D}$. "We sprinkle viruses on a cell and watch how they're taken in," he says. "Now we can track every virus as it enters the cell and follow its fate." Which viruses succeed? Which don't? And what happens as a virus enters a cell? Those are questions Kirchhausen can now ask.

\section{CHALLENGES AHEAD}

Using super-resolution microscopes is not like working an old-fashioned light microscope. "There are no binoculars. Everything is done in the computer," says Kirchhausen."You can see a crude version of what is coming off the microscope, but it takes some time afterwards to render it into something useful," says Mullins. These methods are based on imaging huge numbers of single molecules at a time. That is something that neither the human eye nor brain can make sense of unless powerful software has processed it first.

Most image-processing software has been designed to work with 2D, low-resolution images, explains Mullins. For his work, he collaborated with specialists in processing $3 \mathrm{D}$ images (previously for computer models of individual molecules such as proteins) at the University of California. The video of the neutrophil crawling through a couple of micrometres of tissue was acquired in just 3 minutes in a single experiment that yielded 25 gigabytes of data. A typical high-definition Hollywood film is about one-tenth of that size. Data sharing and storage are becoming a problem.

Hardware is another barrier to using superresolution microscopy. Moerner is using super-resolution microscopes based on both

Betzig and Hell's designs in his lab. His set-ups are complex. A square metre or so of about 30 precisely placed optical components direct the imaging light. The microscopes have to be housed on a vibration-isolating table that is common in physics departments, but not in wet labs.

Such vulnerability is not ideal. Companies including Zeiss and Hell's start-up are designing commercial models that put more compact, fixed versions of these set-ups inside a case. So far the models on the market have not been very good, says Betzig, but that will change.

This move towards

\section{"Until I watched this, I didn't understand the psychology of the cell."}

commercialization does not signal that the fundamental research is slowing down. Hell's dream now, he says, is to map every protein in living tissue in real time at a resolution of damage. Zhuang's dream is similar. One goal in her lab is to follow the waxing and waning of gene expression at the genome scale in individual cells - that would mean imaging the full orchestra of messenger RNA in cells at the same time. This April, she published results from her first attempt, which used a combination of single-molecule imaging and computational savvy to simultaneously detect about 1,000 different kinds of RNA molecules in one cell ${ }^{7}$. Because she is interested in the brain, Zhuang particularly wants to watch how neurons develop, to understand how their different behaviour emerges from gene expression patterns.

Another of Zhuang's goals is to track communication between neurons at their synapses, to watch these cell-to-cell chemical communications just as electrophysiologists 1 nanometre, without causing the tissue any can measure a single neuron's electrical signals. One of her projects is to follow computation in individual neurons. Currently, no one fully understands how a neuron integrates incoming signals and then generates an output. Once that question can be answered at the level of a single cell, observations can be turned towards figuring out the processes of computation in small circuits, and eventually in the whole brain.

Imaging individual cells with these methods is now possible, but it is more difficult to take speedy, single-molecule resolution videos at the tissue level, for example in the brain. Today's systems are held back by the brightness and switching response of existing imaging dyes, and the limited number of colours that can be used at once.

To help them advance the field, Zhuang, Hell, and other imaging researchers are looking to chemistry. "The next frontier is going to be new dye colours," says Luke Lavis, a chemist at the Janelia Research Campus who develops fluorescent imaging tags. Creating dyes that attach to biomolecules in ways that disrupt their function as little as possible - and making a whole rainbow of dyes with colours distinct enough to be separated during image processing will make it possible to view more moving molecules simultaneously. And making dyes brighter, or designing them so that they will not fluoresce until they attach to their target, might stop cells from becoming badly damaged by repeated blasts of high-energy-stimulating light. Lavis is working on it. "When I started out, people would be using synthesis methods reported in 1888, but chemistry has evolved so much since then. Now we can really explore the structures of these dyes," he says.

Watching Mullins explore the subcellular world in his $3 \mathrm{D}$ goggles, it is clear that the details unveiled by today's microscopes would astound researchers such as Abbe and Hooke. Nonetheless, super-resolution imaging is not all about seeing and quantifying cells as collections of molecules. "Until I watched this, I didn't understand the psychology of the cell," says Mullins, only half joking. In this footage, the cell is not an abstract object. It responds to mechanical forces and it pushes back. The cell has a palpable physical presence. And that, compared to all means of watching it before, somehow makes it seem more alive.

Katherine Bourzac is a science journalist based in San Francisco.

1. Klar, T. A., Jakobs, S., Dyba, M., Egner, A. \& Hell, S. W. Proc. Natl Acad. Sci. USA 97, 8206-8210 (2000).

2. Dickson, R. M., Cubitt, A. B., Tsien, R. Y. \& Moerner, W. E. Nature 388, 355-358 (1997)

3. Moerner, W. E. \& Kador, L. Phys. Rev. Lett. 62 , 2535-2538 (1989)

4. Rust, M. J., Bates, M. \& Zhuang, X. Nature Methods 3, 793-796 (2006).

5. Zhong, G. elife 3, e04581 (2014).

6. Fotin, A., et al. Nature 432, 573-579 (2004).

7. Chen, K. H., Boettiger, A. N., Moffitt, J. R., Wang, S. \& Zhuang, X. Science 348, 6233 (2015). 